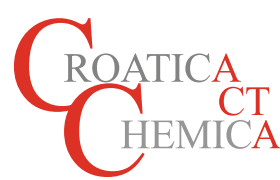

\title{
Model of Reactant Adsorption in dc Polarography
}

\author{
(1) Milivoj Lovrić
}

Abstract: An expanding plane model of polarogram influenced by the reactant adsorption is developed and applied to the Frumkin isotherm. It is shown that the polarogram may consist of the main wave and post-wave that are separated by the maximum and minimum. The origin of these extremes are investigated by the calculation of currents that flow during a single mercury drop.

Keywords: adsorption of reactant, $d c$ polarography, expanding plane model.

\section{INTRODUCTION}

A REACTANT of fast and reversible electrode reaction may be stabilized by the adsorption on the dropping mercury electrode surface. ${ }^{[1,2]}$ If the product is not adsorbed, an additional energy is needed for the reduction of adsorbate and the polarographic wave appears at lower potential than the main wave that corresponds to a simple reaction of dissolved reactant. ${ }^{[3]}$ If the electrode surface is totally covered by the adsorbed reactant before the end of its life time, the main wave appears in front of the adsorption wave. The latter is, for this reason, called the post-wave. A difference between half-wave potentials of the main wave and the post-wave is proportional to the adsorption energy. These two waves may be merged into a single wave if the adsorption is weak. The limiting current of the post-wave increases nonlinearly with the reactant concentration until a certain critical value, after which it stagnates and the main wave develops. ${ }^{[4]}$ In some cases these two waves may be separated by the local current minimum. ${ }^{[5]}$ The separation is explained by the lateral attraction in the adsorbed layer. ${ }^{[6]}$ In the present communication the origin of the minimum is investigated by the expanding plane model. The calculations were inspired by the continuing relevance of $d c$ polarography, ${ }^{[7]}$ but the results are also pertinent to the static mercury drop electrode ${ }^{[5,6]}$ and generally to the chronoamperometry of surface active, electroactive compounds.

\section{THE MODEL}

A reversible redox reaction complicated by the adsorption of reactant on the surface of dropping mercury electrode is considered:

$(\mathrm{Ox})_{\mathrm{ads}} \leftrightarrow \mathrm{Ox}+n \mathrm{e}^{-} \leftrightarrow \mathrm{Red}$

The mass transfer is described by the expanding plane model:

$$
\begin{aligned}
& \partial c_{\mathrm{Ox}} / \partial t=D \partial^{2} c_{\mathrm{Ox}} / \partial x^{2}+(2 x / 3 t) \partial c_{\mathrm{Ox}} / \partial x \\
& \partial c_{\mathrm{Red}} / \partial t=D \partial^{2} c_{\mathrm{Red}} / \partial x^{2}+(2 x / 3 t) \partial c_{\mathrm{Red}} / \partial x \\
& t=0, x \geq 0: \quad c_{\mathrm{Ox}}=c_{\mathrm{Ox}}^{*}, \quad c_{\mathrm{Red}}=0, \Gamma=0 \\
& t>0, x \rightarrow \infty: \quad c_{\mathrm{Ox}} \rightarrow c_{\mathrm{Ox}}^{*}, \quad c_{\mathrm{Red}} \rightarrow 0 \\
& x=0: \quad c_{\mathrm{Ox}, x=0}=c_{\mathrm{Red}, x=0} \exp (\varphi) \\
& \varphi=(n F / R T)\left(E-E^{0}\right) \\
& D\left(\partial c_{\mathrm{Ox}} / \partial x\right)_{x=0}=(I / n F S)+d \Gamma / d t \\
& D\left(\partial c_{\mathrm{Red}} / \partial x\right)_{x=0}=-I / n F S \\
& \beta c_{\mathrm{Ox}, x=0}=\theta(1-\theta)^{-1} \exp (a \theta) \\
& \theta=\Gamma / \Gamma_{\max }
\end{aligned}
$$

The meanings of all symbols are reported in the Table 1.

((c) $\mathrm{EY}$ This work is licensed under a Creative Commons Attribution 4.0 International License. 
Table 1.

\begin{tabular}{|c|l|}
\hline$a$ & Constant of interactions in the adsorbed layer \\
\hline$\beta$ & Adsorption constant of Langmuir isotherm \\
\hline$c_{0 x}$ & Concentration of reactant \\
\hline$c_{\text {Red }}$ & Concentration of product \\
\hline$C_{\text {Ox }}^{*}$ & Concentration of reactant in the bulk of solution \\
\hline$D$ & Diffusion coefficient \\
\hline$d$ & Increment of time \\
\hline$E$ & Electrode potential \\
\hline$E^{0}$ & Standard potential \\
\hline$F$ & Faraday constant \\
\hline$\Gamma$ & Surface concentration of adsorbed reactant \\
\hline$I$ & Current \\
\hline$K$ & Adsorption constant of linear isotherm \\
\hline$M$ & Flow rate of mercury \\
\hline$R$ & Gas constant \\
\hline$S$ & Surface area \\
\hline$T$ & Temperature \\
\hline$t_{d}$ & Drop life time \\
\hline$X$ & Distance from electrode surface \\
\hline & \\
\hline
\end{tabular}

The equations (1)-(10) are solved by the substitution $Y=c_{\mathrm{Ox}}+c_{\mathrm{Red}}$ :

$$
\begin{gathered}
\partial Y / \partial t=D \partial^{2} Y / \partial x^{2}+(2 x / 3 t) \partial Y / \partial x \\
t=0, x \geq 0: \quad Y=c_{\mathrm{Ox}}^{*} \\
t>0, x \rightarrow \infty: \quad Y \rightarrow c_{\mathrm{Ox}}^{*} \\
x=0: \quad Y_{x=0}=c_{\mathrm{Ox}, x=0}(1+\exp (-\varphi)) \\
\beta Y_{x=0} \exp (\varphi) /(1+\exp (\varphi))=\theta(1-\theta)^{-1} \exp (a \theta) \\
D(\partial Y / \partial x)_{x=0}=d \Gamma / d t
\end{gathered}
$$

By the introduction of new variables $z=x t^{2 / 3}$ and $y=t^{7 / 3}$ [Ref. 8] the equations (12) and (17) are transformed as follows:

$$
\begin{aligned}
& \partial Y / \partial y=(3 D / 7) \partial^{2} Y / \partial z^{2} \\
& D(\partial Y / \partial z)_{z=0}=(7 / 3) y^{2 / 7} d \Gamma / d y
\end{aligned}
$$

These equations are solved by the Laplace transformations:

$$
Y_{z=0}=c_{0 x}^{*}-\sqrt{7 / 3 D} \int_{0}^{y} v^{2 / 7}(d \Gamma / d v)(\pi(y-v))^{-1 / 2} d v
$$

The last equation can be combined with the equation (16) to calculate the surface coverage $\theta$ using the Huber method for the numerical solution of integral equations. ${ }^{[9]}$ Firstly, the equation (20) is transformed back to the original variables:

$Y_{x=0}=c_{O x}^{*}-\sqrt{3 / 7 \pi D} \int_{0}^{t} \tau^{-2 / 3}(d \Gamma / d \tau)\left(t^{7 / 3}-\tau^{7 / 3}\right)^{-1 / 2}$.

$(7 / 3) \tau^{4 / 3} d \tau$

Then the time is divided into $m$ increments $(t=m d)$ and it is assumed that the surface concentration $\Gamma$ is constant during the single time increment. So, the first derivation $d \Gamma / d \tau$ can be approximated by the ratio $\left(\Gamma_{i}-\Gamma_{i-1}\right) / d$. This assumption is justified in the Appendix. Under these conditions the integral in eq.(21) is transformed into the sum of increments of $r$ :

$\int_{0}^{t} \tau^{-2 / 3}(d \Gamma / d \tau) t^{-7 / 6}(7 / 3) \tau^{4 / 3}\left(1-(\tau / t)^{7 / 3}\right)^{-1 / 2} d \tau=$

$(m d)^{-7 / 6} \sum_{i=1}^{m}(i d)^{-2 / 3}\left(\left(\Gamma_{i}-\Gamma_{i-1}\right) / d\right) J_{i-1, i}$

$J_{i-1, i}=\int_{(i-1) d}^{i d}(7 / 3) \tau^{4 / 3}\left(1-(\tau / m d)^{7 / 3}\right)^{-1 / 2} d \tau$

$J_{i-1, i}=2 d^{7 / 3} m^{7 / 6} Q_{m, i}$

$Q_{m, i}=\left(m^{7 / 3}-(i-1)^{7 / 3}\right)^{1 / 2}-\left(m^{7 / 3}-i^{7 / 3}\right)^{1 / 2}$

$Y_{x=0}=c_{0 x}^{*}-2(3 / 7 \pi D d)^{1 / 2}\left[m^{-2 / 3} \Gamma_{m} Q_{m, m}-m^{-2 / 3} \Gamma_{m-1} Q_{m, m}+\right.$

$\left.\sum_{i=1}^{m-1} i^{-2 / 3}\left(\Gamma_{i}-\Gamma_{i-1}\right) Q_{m, i}\right]$

$Q_{m, m}=\left(m^{7 / 3}-(m-1)^{7 / 3}\right)^{1 / 2}$

$Q_{m, 1}=m^{7 / 6}-\left(m^{7 / 3}-1\right)^{1 / 2}$

$Q_{1,1}=1$

$\Gamma_{0}=0$

$d=t_{d} / 1000$

$1 \leq m \leq 1000$

Equation (26) is introduced into eq.(16) and the following equation is obtained:

$\left(\theta_{m}-\omega_{m}\right)\left(\theta_{m}-1\right)=b \theta_{m} \exp \left(a \theta_{m}\right)$

$b=f_{1} f_{2} p^{-1} m^{2 / 3} Q_{m, m}^{-1}$

$\omega_{m}=q f_{2} m^{2 / 3} Q_{m, m}^{-1}+\theta_{m-1}-m^{2 / 3} Q_{m, m}^{-1} \sum_{i=1}^{m-1}\left(\theta_{i}-\theta_{i-1}\right) Q_{m, i} / i^{2 / 3}$

$p=\beta \Gamma_{\max } /\left(D t_{d}\right)^{1 / 2}$

$q=c_{\mathrm{Ox}}^{*}\left(D t_{d}\right)^{1 / 2} / \Gamma_{\max }$

$f_{1}=(1+\exp (\varphi)) / \exp (\varphi)$

$f_{2}=(7 \pi / 3000)^{1 / 2} / 2$ 


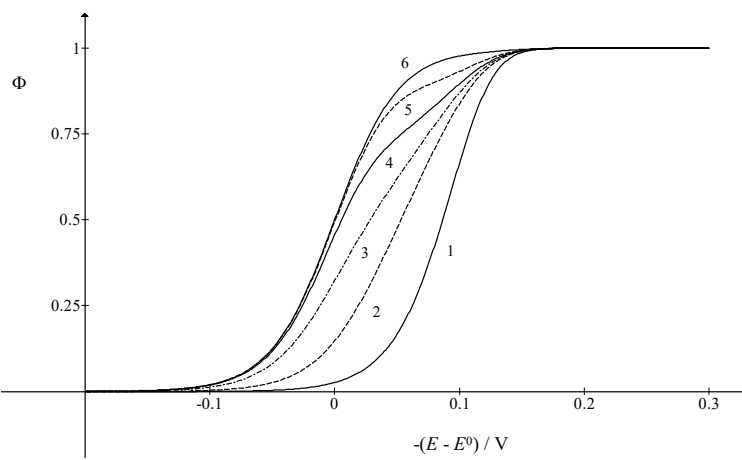

Figure 1. Dimensionless polarograms influenced by the reactant adsorption; $p=100, a=0$ and $q=0.1$ (1), 0.5 (2), $0.6(3), 0.7(4), 0.9(5)$, and 2 (6).

The solution of eq.(33) is obtained by the numerical search. ${ }^{[5]}$ The current is calculated by solving eq.(3):

$$
\begin{aligned}
& \Phi_{m}=(6 / 7) 1000^{-1 / 6}\left[c_{\text {Red }, x=0, m} Q_{m, m} / c_{\mathrm{Ox}}^{*}+\right. \\
& \left.\sum_{j=1}^{m-1} c_{\text {Red }, x=0, j}\left(Q_{m, j}-Q_{m-1, j}\right) / c_{\mathrm{Ox}}^{*}\right] \\
& \Phi_{m}=I_{m}(3 \pi / 7 D)^{1 / 2}\left(0.85 M^{2 / 3} n F c_{\mathrm{Ox}}^{*}\right)^{-1} t_{d}{ }^{-1 / 6} \\
& c_{\text {Red }, x=0, m}=\theta_{m}\left(1-\theta_{m}\right)^{-1} \beta^{-1} \exp \left(a \theta_{m}\right) \exp (-\varphi) \\
& Q_{m-1, j}=\left((m-1)^{7 / 3}-(j-1)^{7 / 3}\right)^{1 / 2}-\left((m-1)^{7 / 3}-j^{7 / 3}\right)^{1 / 2}
\end{aligned}
$$

The current at the end of drop life time is calculated by using $m=1000$, but for the calculation of $I-t$ curves $m$ is changed from 5 to 1000 .

\section{RESULTS AND DISCUSSION}

Theoretical polarograms depend on three dimensionless parameters: the first one is related to the adsorption constant $p=\beta \Gamma_{\max } /\left(D t_{d}\right)^{1 / 2}$, the second is connected with the reactant concentration $q=c_{\mathrm{Ox}}^{*}\left(D t_{d}\right)^{1 / 2} / \Gamma_{\max }$ and the third is the interaction constant $a$. The responses were calculated for $t=t_{d}$, with the potential increment of $1 \mathrm{mV}$ from drop to drop. Hence, the polarograms are sets of current - potential points that are interconnected for better graphical presentation. Some examples are shown in Figure 1. They correspond to the Langmuir isotherm. At low concentration $(q=0.1)$, a post-wave appears with the halfwave potential at $-0.087 \mathrm{~V} v s$. $E^{0}$. The main wave develops gradually, but its limiting current can be recognized only if $q \geq 0.7$. However, the half-wave potentials change from $-0.053 \mathrm{~V}$ (for $q=0.5$ ), over $-0.029 \mathrm{~V}$ for $q=0.6$, to $-0.006 \mathrm{~V}$ for $q=0.7$ and $-0.001 \vee$ for $q=0.9$. If $q=2$ the main wave dominates the response and half-wave potential is equal to $E^{0}$. This potential should not depend on the reactant concentration if the reaction is simple and reversible. ${ }^{[1]}$ So,

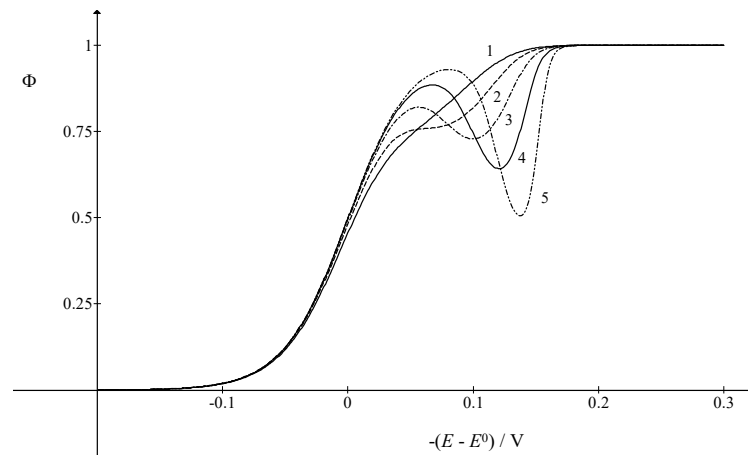

Figure 2. Influence of attraction in the adsorbed layer on the polarograms; $p=100, q=0.7$, and $a=0(1),-1(2),-2(3)$, $-3(4)$, and -4 (5).

the change of $E_{1 / 2}$ towards higher values is good indication of the development of main wave.

The separation between the main wave and the post-wave is significantly enhanced under the influence of lateral attraction within the adsorbate, as can be seen in Figure 2. If $a<-1$ the maximum on the main wave and the minimum at the bottom of post-wave appear. The smaller is $a$, the higher is maximum and the deeper is minimum. Their average value $\left(\Phi_{\max }+\Phi_{\min }\right) / 2$ is close to 0.75 , which is the value of the second inflexion appearing at $-0.05 \mathrm{~V} v$ s. $E^{0}$ in the polarogram 2 that corresponds to $a=-1$. It seems that this current depends on the parameter $q$. However, the average potential $\left(E_{\max }+E_{\min }\right) / 2$ increases from $-0.08 \mathrm{~V}$, for $a=-2$, to $-0.11 \mathrm{~V}$ for $a=-4$. If the interactions between adsorbed molecules are repulsive, the apparent adsorption constant is diminished with the increasing surface coverage and the separation between the main wave and post wave vanishes.

Figure 3 shows the influence of parameter $p$ on polarograms. If $D=9 \times 10^{-6} \mathrm{~cm}^{2} / \mathrm{s}, t_{d}=1 \mathrm{~s}$ and $\Gamma_{\max }=10^{-9}$ $\mathrm{mol} / \mathrm{cm}^{2}$, the value $p=100$ corresponds to the adsorption constant $\beta=3 \times 10^{8} \mathrm{~cm}^{3} / \mathrm{mol}$, which means that the adsorption is rather strong. ${ }^{[5,10,11]}$ More realistic is the value

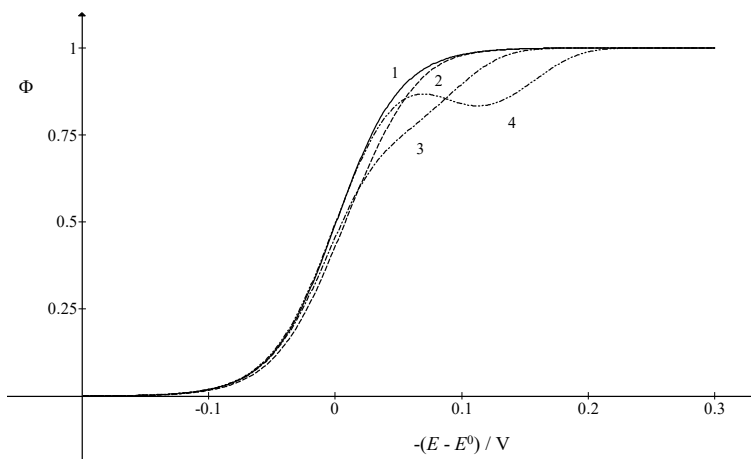

Figure 3. Influence of dimensionless adsorption constant on the polarograms; $q=0.7, a=0$, and $p=1(1), 10(2), 100$ (3), and 1000 (4). 


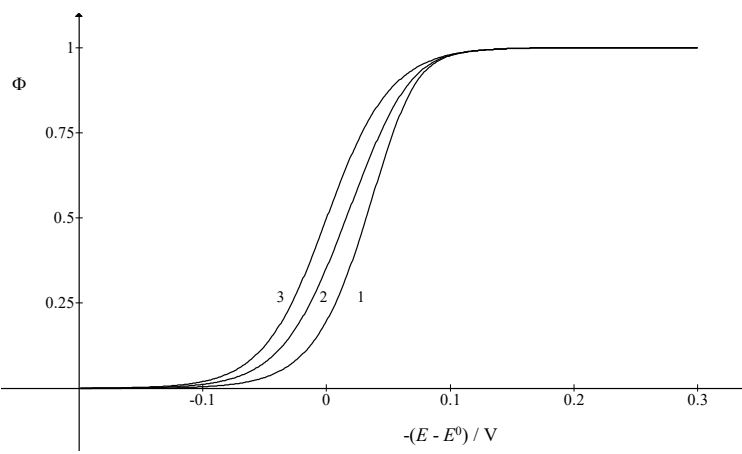

Figure 4. Polarograms influenced by the weak adsorption that follows Langmuir isotherm; $p=10, a=0$, and $q=0.1$ (1), 0.5 (2), and 2 (3).

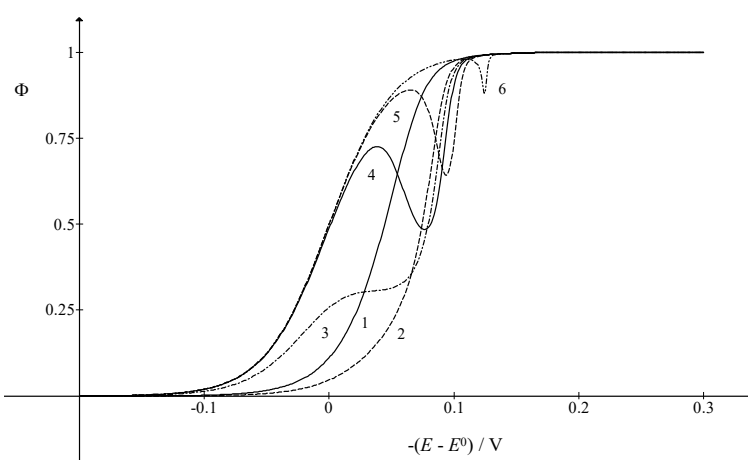

Figure 5. Polarograms influenced by the adsorption that follows Frumkin isotherm; $p=10, a=-4$, and $q=0.1$ (1), 0.5 (2), 0.6 (3), 0.7 (4), 0.9 (5), and 2 (6).

$p=10$, but in this case the main wave and the post-wave are merged. The opposite effect appears if $p=1000$ : the two waves are separated by the maximum at $-0.070 \mathrm{~V} v$ s. $E^{0}$ and the minimum at $-0.113 \mathrm{~V}$. This result indicates that the maxima and minima are consequences of the product $\beta \exp (-a \theta)$ in Frumkin isotherm. Figures 4 and 5 confirm this conclusion. They were calculated assuming weaker adsorptions following Langmuir and Frumkin isotherms, respectively. In Figure 4 one can notice that the variation of reactant concentration does not change the form of response, but only the half-wave potential that increases from $-0.032 \vee v s$. $E^{0}$ for $q=0.1$, over $-0.016 \vee$ for $q=0.5$, to $0 \mathrm{~V}$ for $q=2$. In the case of Frumkin isotherm the changes are more dramatic. Firstly, the exponential term increases the apparent adsorption constant even under the conditions of post-wave. For $q=0.1$ the half-wave potential is $-0.044 \mathrm{~V}$ vs. $E^{0}$, which is lower than in Figure 4 , and for $q=0.5$ it is $-0.075 \mathrm{~V}$ because the surface concentration is close to saturation. At $q=0.6$ the main wave appears and its limiting current is well defined. As the reactant concentration increases, the response split in two waves separated by maxima and minima. The half-wave potential of the main wave is independent of the reactant concentration, while the half-wave potential of post-wave is lower if the concentration is higher. This is because the forces of lateral attraction within the adsorbed layer require an additional energy for the reduction of stabilized reactant.

To explain the origin of maxima and minima, the currents flowing during the life time of a single drop were calculated at various potentials. Figure 6 shows the chosen polarogram. It is characterized by the maximum at $-0.032 \mathrm{~V}$ and the minimum at $-0.054 \mathrm{~V} v s$. $E^{0}$. Figure 7 shows the $I-t$ curves and the corresponding surface coverages are

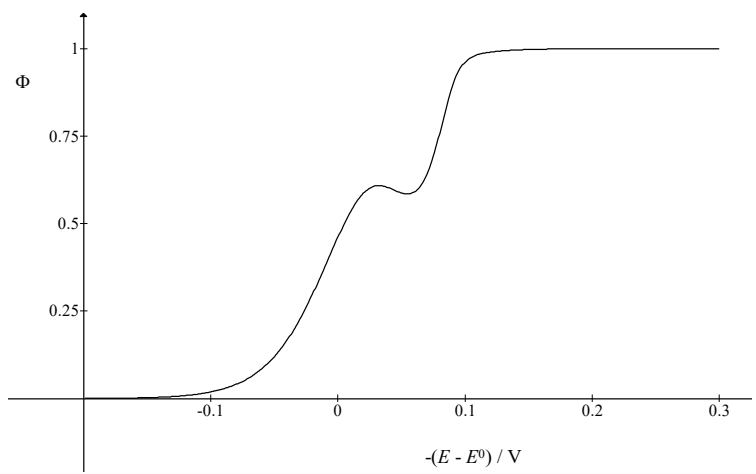

Figure 6. Polarogram with the maximum and minimum; $p=10, q=0.7$, and $a=-3$.

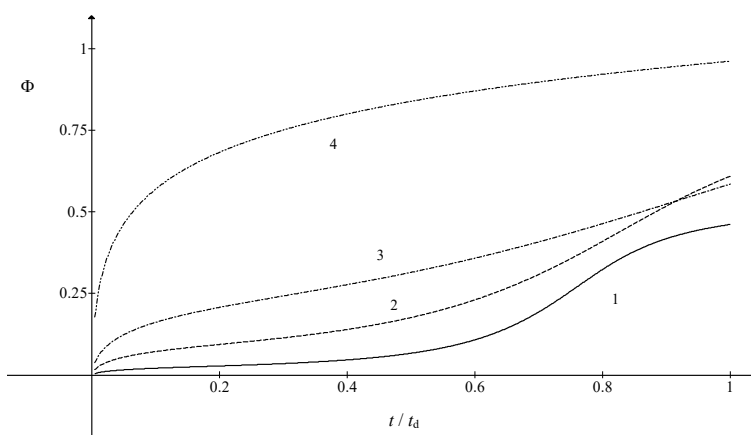

Figure 7. The currents flowing at a single drop; $\left(E-E^{0}\right) / \mathrm{V}=0$ (1), -0.032 (2), -0.054 (3), and -0.1 (4). All other data are as in Figure 6.

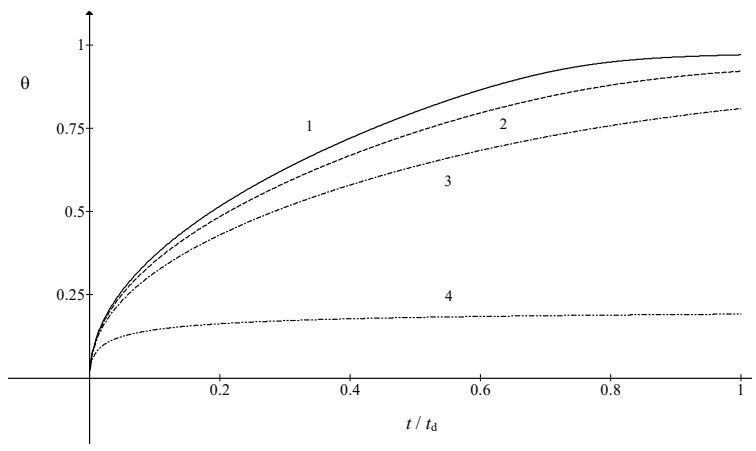

Figure 8. Surface coverages at various potentials; $\left(E-E^{0}\right) / \mathrm{V}=0$ (1), -0.032 (2), -0.054 (3), and -0.1 (4). All other data are as in Figure 6. 
shown in Figure 8. At zero volts versus $E^{0}$ the flux of reactant is divided between the adsorption and the reduction. The coverage $\theta$ increases to 0.8 in the first half of the drop life time and to 0.97 in the other half. Consequently, the current is very low in the beginning of the drop life time and increases sigmoidally in the second half of this time. It is important to realise that $\theta$ depends on electrode potential as well as on the reactant concentration and the time. At $-0.032 \mathrm{~V}$ the coverage is growing slower than at $0 \mathrm{~V}$ : $\theta=0.74$ at $t=t_{d} / 2$ and $\theta=0.92$ at $t=t_{d}$. For this reason the current is higher throughout the drop life time. At twenty two millivolts lower potential $\theta$ decreases to 0.635 and 0.81 at $t=t_{d} / 2$ and $t=t_{d}$, respectively. The corresponding current is higher from the beginning of the drop life time, but without the sigmoidal increasing at the end of this time. This is the reason why the curves 2 and 3 in Figure 7 are intersecting and why the current at $t=t_{d}$ is higher at $-0.032 \mathrm{~V}$ than at $-0.054 \mathrm{~V}$. In this way the maximum and minimum in the polarogram appear. The curves 4 in Figures 7 and 8 correspond to the post-wave. The coverage is lower than 0.2 and the flux is consumed by the reduction almost entirely.

The differences $\Phi_{\max }-\Phi_{\min }$ and $E_{\max }-E_{\min }$ are very sensitive to the parameters $p, q$ and $a$. So, these parameters can be estimated by fitting the experimental results with the described model.

The model is developed for the anion-induced adsorption of amalgam forming metal ions. Usually, only the neutral complex is adsorbed, ${ }^{[6]}$ but the adsorption of negatively or positively charged ions is also possible. In the latter case the adsorption constant may increase as the electrode potential becomes more negative. An example is shown in Figure 9. It is calculated for the potential dependent parameter $p=12-10\left(E-E^{0}\right)$. This function is chosen arbitrarily. As the starting potential is $0.2 \mathrm{~V} v s$. $E^{0}$, the parameter $p$ increases from 10 to 15 . The response is similar to Figure 6 , but the maximum is higher than the one corresponding to $p=10$ and lower than the one corresponding to $p=15$.

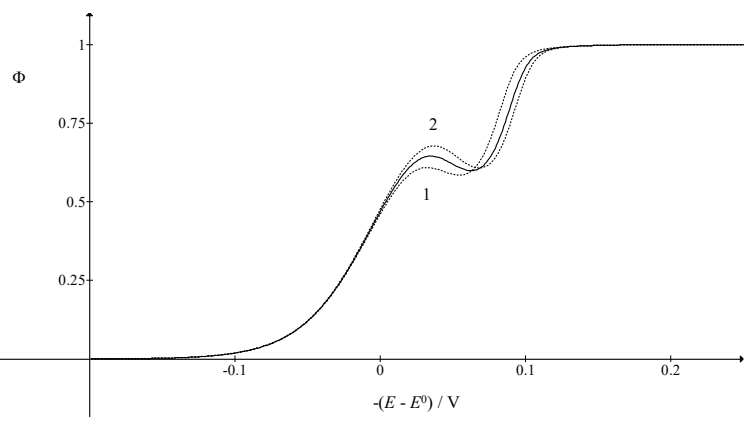

Figure 9. Dimensionless polarogram calculated for the parameter $p=12-10\left(E-E^{0}\right)$ (full line). Polarograms that are calculated for $p=10$ (1) and 15 (2) are shown by dotted lines. Other parameters are as in Figure 6.
Under the influence of some electro-inactive surface active substance the equation (10) must be replaced by the equation

$$
\beta c_{\mathrm{ox}, x=0} \exp (-a \theta)=\theta /\left(1-\theta-\theta_{\mathrm{SAS}}\right)
$$

For the sake of simplicity, it is assumed that the diffusion of SAS can be neglected. Also, no interactions between adsorbed SAS molecules are assumed:

$\beta_{\mathrm{SAS}} c_{\mathrm{SAS}}^{*}=\theta_{\mathrm{SAS}} /\left(1-\theta-\theta_{\mathrm{SAS}}\right)$

Under this condition the equation (33) appears in the form:

$(\theta-\omega)(\theta-1)=b \theta \exp (a \theta)\left(1+\beta_{\mathrm{SAS}} c_{\mathrm{SAS}}^{*}\right)$

The effects of SAS are shown in Figure 10. It is calculated for $p=10, a=-3$ and $\beta_{S A S} c_{S A S}^{*}=1$. In the presence of SAS the maximum appears at the concentration parameter $q=0.9$, which is higher than in Figure 6.

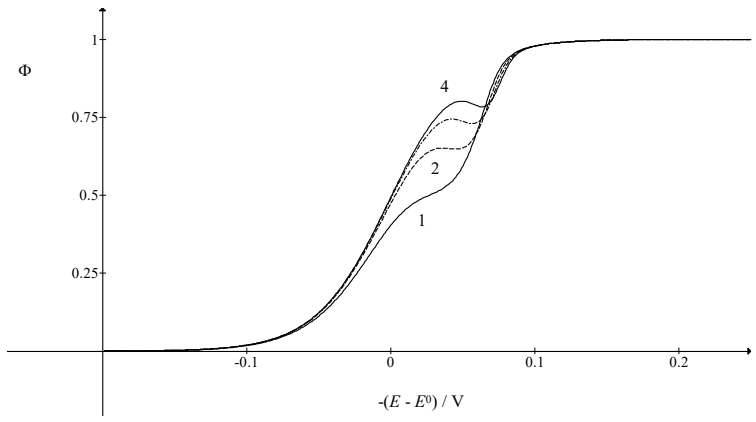

Figure 10. Polarograms influenced by the surface active substance; $\beta_{\text {SAS }} c_{S A S}^{*}=1$ and $q=0.7$ (1), 0.8 (2), 0.9 (3) and 1 (4). All other data are as in Figure 6.

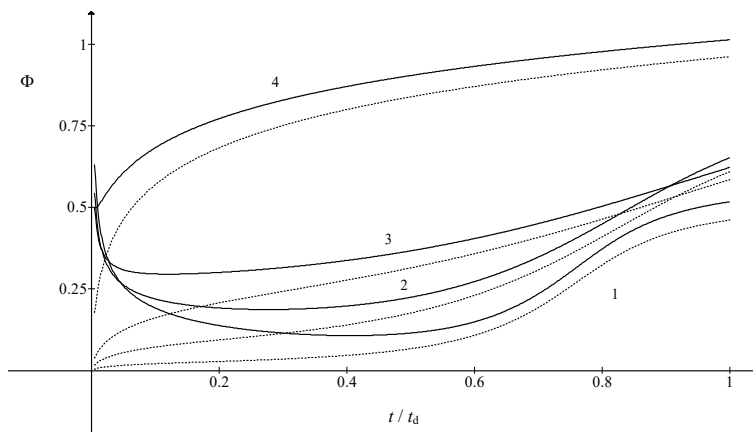

Figure 11. Sum of capacitive and faradaic currents during the drop life-time (full lines). $C_{\theta=0}=50 \mu \mathrm{F} / \mathrm{cm}^{2}, C_{\theta=1}=25$ $\mu \mathrm{F} / \mathrm{cm}^{2}, E_{m}-E^{0}=-0.2 \mathrm{~V}, c_{\mathrm{Ox}}^{*}=2.3 \times 10^{-7} \mathrm{~mol} / \mathrm{cm}^{3}$ and $E-E^{0} / \mathrm{V}=0(1),-0.032$ (2), -0.054 (3) and -0.1 (4). All other parameters are as in Figure 7. Faradaic components of currents are shown by dotted lines. 
Capacitive currents on the dropping mercury electrode decreases with $t^{1 / 3}:$ [1]

$$
\begin{aligned}
& I_{C}=2 C\left(E-E_{m}\right) S_{\max }\left(t_{d} / t\right)^{1 / 3} / 3 t_{d} \\
& S_{\max }=0.85 \mathrm{M}^{2 / 3} t_{d}^{2 / 3}
\end{aligned}
$$

Under the influence of reactant adsorption, the capacity depends on the surface coverage:

$$
C=C_{\theta=0}(1-\theta)+C_{\theta=1} \theta
$$

If it is assumed that the adsorption does not change the zero charge potential, the dimensionless capacitive current is given by the following equation:

$$
\begin{aligned}
& \Phi_{C}=\left(C_{\theta=0} / n F C_{O x}^{*}\right) \sqrt{3 \pi / 7 D t_{d}}\left[2\left(t_{d} / t\right)^{1 / 3}(1+\right. \\
& \left.\left.\Delta C \theta / C_{\theta=0}\right) / 3+\Delta C\left(t / t_{d}\right)^{2 / 3}(d \theta / d t) / C_{\theta=0}\right]\left(E-E_{m}\right) \\
& \Delta C=C_{\theta=1}-C_{\theta=0} \\
& \Phi_{C}=I_{C}\left(n F S_{\max } C_{O x}^{*}\right)^{-1} \sqrt{3 \pi t_{d} / 7 D}
\end{aligned}
$$

This normalization is applied to compare the capacitive current with the faradaic one that is defined by eq. (41). Figure 11 show the sum of these two currents during the life time of a single drop at various potentials. The parameters of electrode reaction are the same as in Figure 7. So, the faradaic currents presented in the latter figure are shown in Figure 11 by dotted curves. Assuming that $\Gamma_{\max }=10^{-9} \mathrm{~mol} / \mathrm{cm}^{2}$, the bulk reactant concentration that corresponds to $q=0.7$ is $2.3 \times 10^{-4} \mathrm{~mol} / \mathrm{L}$. One can notice that the influence of capacitive current is the highest at the beginning of the drop life-time, but at its end this current is almost independent of the potential. So, in the tast polarography the response similar to the curve shown in Figure 6 can be obtained.

\section{CONCLUSIONS}

Polarograms influenced by the reactant adsorption may consist of the main wave and post-wave, but these two components are poorly separated if the adsorption is not very strong. Under the influence of lateral attraction in the adsorbed layer the apparent adsorption constant increases and the polarogram with maximum and minimum may appear. These extremes separate the main wave from the post-wave. The limiting current of the main wave that is parallel with the limiting current of post-wave is a special case of the response with the maximum and minimum.

\section{APPENDIX}

Adsorption of electro-inactive compound on the surface of stationary planar electrode is considered:

$$
\begin{gathered}
\mathrm{A} \leftrightarrow \mathrm{A}_{\mathrm{ads}} \\
\partial c_{\mathrm{A}} / \partial t=D \partial^{2} c_{\mathrm{A}} / \partial x^{2} \\
t=0, x \geq 0: \quad c_{\mathrm{A}}=c_{\mathrm{A}}^{*}, \quad \Gamma=0 \\
t>0, x \rightarrow \infty: \quad c_{\mathrm{A}} \rightarrow c_{\mathrm{A}}^{*} \\
x=0: \quad c_{\mathrm{A}, x=0}=K \Gamma \\
D\left(\partial c_{\mathrm{A}} / \partial x\right)_{x=0}=d \Gamma / d t
\end{gathered}
$$

Using Laplace transformations one obtains:

$\mathcal{L} c_{\mathrm{A}, x=0}=c_{\mathrm{A}}^{*} / s-D^{-1 / 2} s^{-1 / 2} \mathcal{L}(d \Gamma / d t)$

a) Analytical solution

Considering the conditions (A3) and (A5) one can write:

$\mathcal{L}(d \Gamma / d t)=s \mathcal{L} \Gamma$

$\mathcal{L} c_{\mathrm{A}, x=0}=K \mathcal{L} \Gamma$

The last two equations are introduced into eq.(A7) and the following solution is obtained:

$\Gamma=c_{\mathrm{A}}^{*} K^{-1}\left[1-\exp \left(K^{2} D t\right) \operatorname{erf} c(K \sqrt{D t})\right]$

b) The first integral equation

Using eq. (A8) the inverse Laplace transformation of the last term in eq.(A7) can be written as:

$\mathcal{L}^{-1}\left\{D^{-1 / 2} s s^{-1 / 2} \mathcal{L} \Gamma\right\}=D^{-1 / 2} \frac{\partial}{\partial t} \int_{0}^{t} \Gamma(\pi(t-\tau))^{-1 / 2} d \tau$

If a time is divided in $m$ increments $(t=m d)$, the first derivative of the integral in eq.(A11) can be approximated by the following ratio:

$$
\begin{aligned}
& \frac{\partial}{\partial t} \int_{0}^{t} \Gamma(\pi(t-\tau))^{-1 / 2} d \tau=d^{-1}\left\{\int_{0}^{m d} \Gamma(\pi(m d-\tau))^{-1 / 2} d \tau-\right. \\
& \left.\int_{0}^{(m-1) d} \Gamma(\pi((m-1) d-\tau))^{-1 / 2} d \tau\right\}
\end{aligned}
$$

Then it is assumed that $\Gamma$ is constant within a single time increment and a recursive formula for the surface concentration is obtained:

$$
\begin{aligned}
& \left(K+2(\pi D d)^{-1 / 2}\right) \Gamma_{m}=c_{\mathrm{A}}^{*}-2(\pi D d)^{-1 / 2} \sum_{i=1}^{m-1} \Gamma_{i}\left(Q_{m-i+1}-Q_{m-i}\right) \\
& Q_{k}=k^{1 / 2}-(k-1)^{1 / 2}
\end{aligned}
$$

\section{c) The second integral equation}

The inverse Laplace transformation of eq.(A7) gives:

$c_{\mathrm{A}, x=0}=c_{\mathrm{A}}^{*}-D^{-1 / 2} \int_{0}^{t}(d \Gamma / d \tau)(\pi(t-\tau))^{-1 / 2} d \tau$

If the time is divided into increments, it can be assumed that the flux of $A$ is constant during the single time 
increment:

$(d \Gamma / d \tau)_{i, i-1}=\left(\Gamma_{i}-\Gamma_{i-1}\right) / d$

Hence, the integral in eq.(A15) is approximated by the sum of finite flux increments:

$D^{-1 / 2} \pi^{-1 / 2} \int_{0}^{t}(d \Gamma / d \tau)(t-\tau)^{-1 / 2} d \tau=$

$d^{-1}(\pi D)^{-1 / 2} \sum_{i=1}^{m}\left(\Gamma_{i}-\Gamma_{i-1}\right) \int_{(i-1) d}^{i d}(m d-\tau)^{-1 / 2} d \tau$

$\Gamma_{0}=0$

Under these assumptions the following recursive formula is obtained:

$$
\begin{aligned}
& \left(K+2(\pi D d)^{-1 / 2}\right) \Gamma_{m}=c_{\mathrm{A}}^{*}+2(\pi D d)^{-1 / 2} \Gamma_{m-1}- \\
& 2(\pi D d)^{-1 / 2} \sum_{i=1}^{m-1}\left(\Gamma_{i}-\Gamma_{i-1}\right) Q_{m-i+1}
\end{aligned}
$$

Equations (A10), (A13) and (A19) were compared using the time increment $d=t_{d} / 1000$. Table 2 shows relative errors $R_{1}=100 \times$ (eq. $(\mathrm{A} 13)-$ eq. $\left.(\mathrm{A} 10)\right) /$ eq. $(\mathrm{A} 10)$ and $R_{2}=100 \times$ (eq. (A19) - eq. $\left.(\mathrm{A} 10)\right) /$ eq. $(\mathrm{A} 10)$ that were

Table 2.

\begin{tabular}{ccc}
\hline$t / t_{d}$ & $R_{1} / \%$ & $R_{2} / \%$ \\
\hline 0.2 & -0.1122 & -0.1122 \\
0.4 & -0.0535 & -0.0535 \\
0.6 & -0.0344 & -0.0344 \\
0.8 & -0.0251 & -0.0251 \\
1 & -0.0196 & -0.0196 \\
\hline
\end{tabular}

calculated for $K=100 \mathrm{~cm}^{-1}, D=9 \times 10^{-6} \mathrm{~cm}^{2} / \mathrm{s}$ and $t_{d}=1 \mathrm{~s}$. Equations (A13) and (A19) give identical results. This is proof that eq.(22) is correct.

\section{REFERENCES}

[1] J. Heyrovsky, J. Kuta, Principles of polarography, Academic Press, New York, 1966, p. 287. https://doi.org/10.1016/B978-0-12-346650-1.50020-5

[2] R. Brdička, Z. Elektrochem. 1942, 48, 278.

[3] T. Fujinaga, K. Izutsu, K. Yamashita, Rev. Polarog. 1965, 13, 52-59.

https://doi.org/10.5189/revpolarography.13.52

[4] Z. Galus, Fundamentals of electrochemical analysis, II ed., Ellis Horwood and Polish Scientific Publishers, New York, Warsaw, 1994, p. 481.

[5] M. Zelić, M. Lovrić, Electrochim. Acta 1990, 35, 1701-1706.

https://doi.org/10.1016/0013-4686(90)87068-D

[6] M. Lovrić, Š. Komorsky-Lovrić, Langmuir 1995, 11, 1784-1790.

[7] P. Zuman, Acta Chim. Slov. 2009, 56, 18-29.

[8] R. S. Subrahmanya, Canad. J. Chem. 1962, 40, 296-300. https://doi.org/10.1139/v62-048

[9] L. K. Bieniasz, Modelling electrochemical experiments by the integral equation method, Springer, Berlin, 2015. https://doi.org/10.1007/978-3-662-44882-3

[10] D. Gugała-Fekner, Croat. Chem. Acta 2016, 89, 25. https://doi.org/10.5562/cca2688

[11] D. Gugała-Fekner, Chem. Monthly 2018, 149, 13571365. https://doi.org/10.1007/s00706-018-2183-1 